\title{
Transitional and temporary objects in the Jupiter Trojan area
}

\author{
O. Karlsson ${ }^{\star}$ \\ Astronomiska Observatoriet, Uppsala Universitet, Box 515, 75120 Uppsala, Sweden \\ Received 4 March 2003/ Accepted 4 September 2003

\begin{abstract}
I have integrated 1233 objects with semimajor axes between 4.6 and 5.8 AU 1000 years back and forward in time. A search for Jupiter horseshoe Trojans in transition from tadpole orbits to non-Trojan orbits and objects in temporary horseshoe orbits was made. A total of 13 objects (5 comets, 3 asteroids and 5 Trojans) were found. In a second group, among 337 new Trojans discovered after the primary investigation, 7 transitional candidates were found. Two objects, $2000 \mathrm{HR}_{24}$ and $2002 \mathrm{GP}_{31}$, are falsely identified as Trojans. No likely transitional objects were found in the first object set, but all of them have at least temporary captures in orbit types associated with Jupiter's 1:1 mean motion resonance. Trojans in the first set have short observational arcs which can explain the low probability for a Trojan past. Trojans in the second set have longer arcs and 5 of them are good candidates for being in transition from the Trojan area.
\end{abstract}

Key words. comets: general - minor planets, asteroids

\section{Introduction}

Until 1997-1998 most of the Jupiter Trojans were discovered in surveys covering small areas around the Lagrangian point (LP) four $\left(\mathrm{L}_{4}\right)$ or five $\left(\mathrm{L}_{5}\right)$. The discovered Trojans were among the most stable ones.

The first of these surveys was the Palomar-Leiden Survey (designated PLS) in 1960 close to $\mathrm{L}_{4}$ (Van Houten et al. 1970b), see Fig. 1. In 1965, Van Houten et al. (1970a) obtained additional positions of new Trojans for an estimation of the size of the Trojan cloud. Follow-up studies were made at three occasions, in 1971 (Marsden 1991) of $\mathrm{L}_{5}$ (designated $\mathrm{T}-1$ ), in 1973 (Marsden 1989) of $\mathrm{L}_{4}$ (designated T-2) and in 1977 (Marsden 1987) of $\mathrm{L}_{5}$ (designated T-3). In 1985, 1988 and 1989 Shoemaker et al. (1989) searched both $\mathrm{L}_{4}$ and $\mathrm{L}_{5}$. The Uppsala-DLR Trojan Survey (Lagerkvist et al. 2000, 2002) of $\mathrm{L}_{4}$ were made in 1996 (600 square degrees), 1997 (600 square degrees) and 1998 (1000 square degrees). Since about 1997 several large surveys for Near Earth Objects (NEO) have been running, and cover most of the northern sky each year. One byproduct of these surveys are new Trojans, many currently distant from the LPs. The further away from a LP a Trojan is found, the more probable it is to be loosely bound to the LP. It is therefore relevant to start looking for Trojans in a transitional phase between the $\mathrm{L}_{4}$ or $\mathrm{L}_{5}$ clouds and the surrounding space.

From several numerical studies (e.g. Marzari et al. 1995; Levison et al. 1997; Marzari \& Scholl 2002) it is clear that transitional objects moving from tadpole orbits to non-Trojan orbits must exist. The transition time ranges from a few

^ e-mail: 0la.Karlsson@astro.uu.se

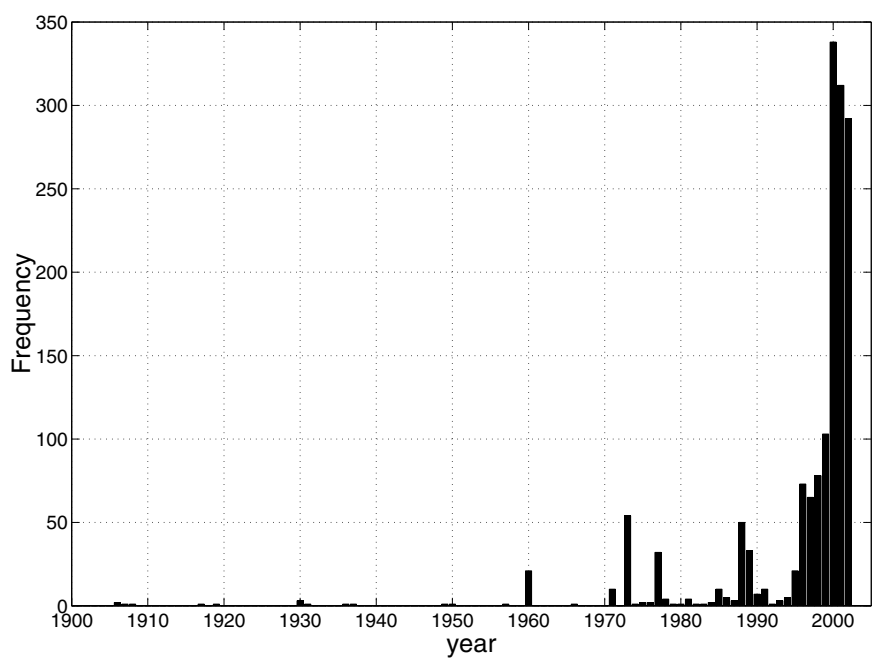

Fig. 1. Trojan discoveries based on their designation. Before 1997-1998 most discoveries came from dedicated surveys close to $\mathrm{L}_{4}$ or $\mathrm{L}_{5}$. In 1906, (588) Achilles and (617) Patroclus, the first Trojans in $L_{4}$ and $L_{5}$ respectively, can be seen. Since the year 2000 the number of discoveries has been dropping. This can be due to that the Trojan completion magnitude is close to the detection limit of the survey telescopes. However, since the orbital period for the Trojans is almost 12 years, new Trojans close to aphelion today and slightly too faint for detection will appear in the years to come. The $\mathrm{L}_{4}$ group is now entering the southern sky where the survey coverage is at best poor. Positions from the surveys of 1965 and 1998 are not in the MPC files, and therefore not present in the histogram.

libration periods to hundreds of thousands of years, but is generally some thousand or tens of thousand years long. 
Table 1. Initial orbital elements for the selected objects with clones checked against known observations. The Tisserand parameter is referring to Jupiter and its reference plane. For $2000 \mathrm{HR}_{24}$ the listed LP is not relevant, see Sect. 3.3. The arc lengths are: $\mathrm{y}=$ years, o = oppositions and $\mathrm{d}=$ days. The orbit types are: $\mathrm{N}=$ Non-Trojan, $\mathrm{TL}=$ Trojan-like, $\mathrm{HL}=$ Horseshoe-like and $\mathrm{H}=$ Horseshoe. For K00E37J the Trojan future is a skew horseshoe orbits.

\begin{tabular}{l|rrrc|ccc|ccc}
\hline \hline Name & $a$ & $e$ & $i$ & Epoch & Tisserand & LP & Arc & Trojan past & Trojan future & Current orbit \\
\hline 53P & 5.39 & 0.552 & 6.6 & 2452922.0 & 2.65 & - & $39 \mathrm{y}$ & - & $6 \%$ & $\mathrm{HL}$ \\
PJ97V010 & 4.94 & 0.331 & 12.1 & 2452200.5 & 2.85 & - & $92 \mathrm{~d}$ & $12 \%$ & $7 \%$ & $\mathrm{HL}$ \\
PJ98U040 & 5.58 & 0.310 & 31.5 & 2452200.5 & 2.61 & - & $386 \mathrm{~d}$ & $2 \%$ & $18 \%$ & $\mathrm{~N}$ \\
PJ99W07J & 4.63 & 0.316 & 3.0 & 2452200.5 & 2.91 & - & $2.5 \mathrm{y}$ & $3 \%$ & $8 \%$ & $\mathrm{~N}$ \\
PK00Y030 & 5.04 & 0.197 & 2.3 & 2452200.5 & 2.96 & - & $354 \mathrm{~d}$ & $0 \%$ & $2 \%$ & $\mathrm{H}$ \\
6144 & 4.75 & 0.366 & 5.9 & 2452400.5 & 2.87 & - & $5 \mathrm{o}$ & $1 \%$ & $15 \%$ & $\mathrm{~N}$ \\
32511 & 5.06 & 0.427 & 8.9 & 2452400.5 & 2.78 & - & $4 \mathrm{o}$ & $3 \%$ & $8 \%$ & $\mathrm{~N}$ \\
J95S24G & 4.96 & 0.172 & 3.1 & 2449980.5 & - & $\mathrm{L}_{4}$ & $11 \mathrm{~d}$ & $17 \%$ & $6 \%$ & $\mathrm{H}$ \\
J96A10V & 4.90 & 0.141 & 19.6 & 2450100.5 & - & $\mathrm{L}_{5}$ & $14 \mathrm{~d}$ & $6 \%$ & $19 \%$ & $\mathrm{TL}$ \\
J97T02W & 5.47 & 0.260 & 11.0 & 2450720.5 & - & $\mathrm{L}_{4}$ & $2 \mathrm{~d}$ & $10 \%$ & $7 \%$ & $\mathrm{H}$ \\
K00E37J & 4.69 & 0.704 & 10.2 & 2452400.5 & 2.44 & - & $2 \mathrm{o}$ & $2 \%$ & $(15) \%$ & $\mathrm{~N}$ \\
K00H24R & 4.96 & 0.175 & 15.5 & 2452400.5 & 2.89 & $\left(\mathrm{~L}_{5}\right)$ & $2 \mathrm{o}$ & $0 \%$ & $1 \%$ & $\mathrm{~N}$ \\
K00QN3V & 5.13 & 0.248 & 4.8 & 2451780.5 & - & $\mathrm{L}_{5}$ & $3 \mathrm{~d}$ & $19 \%$ & $6 \%$ & $\mathrm{H}$ \\
\hline
\end{tabular}

This time is much less than the time spent in a tadpole orbit. Thus the expected number of transition objects found at any given time is very low.

In this paper, I show that there are some Trojans present today that are candidates for having non-tadpole orbits. Also there are some known comets that have, or have had Trojantype orbits in the near past, the present or the near future.

\section{Numerical integration}

In asteroid and comet files issued from the Minor Planet Center (MPC) at the end of February 2002, all objects (1215 Trojans, 10 asteroids and 8 comets) with semimajor axes between 4.6 and 5.8 AU at the given epoch were selected and integrated 1000 years back and forward in time with the rmvs3 integrator, in the SWIFT integrator package (Levison \& Duncan 1994), based on the Mixed Variable Symplectic method (Wisdom \& Holman 1991). The main integration time step (TS) was 10 days. All the eight major planets were included (DE406/LE406). Perturbations from the terrestrial planets are insignificant in this case, but they were included for easy reference purposes in the analysis if necessary. The time span was selected from a practical point of view.

The rmvs3 integrator uses two subareas around the planets where the time step length can be changed. The integrator was compiled with TS/40 within 3.5 Hillradii and TS/160 within 1 Hillradius.

As a check of the length of the TS a loosely bound tadpole Trojan (with passages relatively close to Jupiter each 150 years) was used. Two integrations were made with the same conditions as in the main integration, first with only the Trojan and secondly with the Trojan plus four other objects with frequent encounters with Jupiter. As a result of the extra approaches to Jupiter in the second integration, the two subareas with shorter TS were more frequently visited. The difference in the evolution of the semimajor axis for the Trojan in the two integrations were smaller than the seventh decimal (the number of decimal given by MPC for the orbital elements) during a few thousand years, thus indicating that the TS is small enough for the \pm 1000 year time span in the main integration.

Most of the 1233 selected objects were ordinary tadpole Trojans and therefore not interesting for this paper. 27 of the objects (11 Trojans, 9 asteroids and 7 comets) were picked out for closer study since they showed transitional or close to transitional behaviour during the time span. A. Gnädig provided me with orbit determinations, including errors for the orbital elements, for the unnumbered asteroids and Trojans. For the remaining objects, orbital elements from MPC were used.

For each of these remaining 27 objects, 99 additional fictitious orbits were added in order to study the dynamical stability of the orbits. The new orbits were checked so that they fitted within $2^{\prime \prime}$ of reported positions in the MPC files. The main integration was then redone for each of the 27 object batches from the epoch given in each orbit solution. No non-gravitational forces were used for the comets.

Among the 27 objects, 13 objects ( 5 Trojans, 3 asteroids and 5 comets) turned out to have at least one horseshoe-like or tadpole-like period for more than half of the object's clones during the time span of 2000 years. Of the 5 Trojans, 2 are in the $L_{4}$ region and 3 are in the $L_{5}$ region. Data of these objects are listed in Table 1.

In October 2002, 337 more Trojans were known than in February 2002. The nominal orbits of these new Trojans were like the February 2002 objects integrated 1000 years back and forward in time, with the same parameter settings. Of these, 7 had orbits with transitional behaviour (listed in Table 2). Of the 7 objects, 1 had a larger semimajor axis than Jupiter, while the other 6 had smaller semimajor axes than Jupiter. As done for the objects in Table 1, 99 additional fictitious orbits were added for each Trojan. These orbits were not checked against the positions, instead they were randomly generated within a 6-dimensional box (of the same size for each Trojan) with the nominal orbit at the center. The errors used (size of the box) were twice the error used for generating random orbits for the Trojan $1996 \mathrm{AV}_{10}$. The error of $1996 \mathrm{AV}_{10}$ were chosen because it is a Trojan and the length of the observational arc is of 
Table 2. Initial orbital elements for specially selected Trojans with no check against known observations. For $2002 \mathrm{GP}_{31}$ the listed LP is not relevant, see Sect. 3.4. The arc lengths are $\mathrm{d}=$ days. The orbit types are: $\mathrm{N}=$ Non-Trojan, $\mathrm{H}=$ Horseshoe and $\mathrm{T}=\mathrm{Tadpole}$. Orbital elements are from October 2002.

\begin{tabular}{|c|c|c|c|c|c|c|c|c|c|}
\hline Name & $\bar{a}$ & $\bar{e}$ & $\bar{i}$ & Epoch & $\overline{\mathrm{LPP}}$ & $\overline{\text { Arc }}$ & Trojan past & Trojan future & Current Orbit \\
\hline K01RA0G & 4.75 & 0.044 & 7.8 & 2452140.5 & $\mathrm{~L}_{5}$ & $47 d$ & $3 \%$ & $4 \%$ & $\mathrm{H}$ \\
\hline K01V03J & 4.92 & 0.153 & 4.8 & 2452220.5 & $\mathrm{~L}_{5}$ & $12 d$ & $18 \%$ & $5 \%$ & $\mathrm{H}$ \\
\hline K02EB2X & 4.97 & 0.183 & 6.5 & 2452320.5 & $\mathrm{~L}_{4}$ & $20 \mathrm{~d}$ & $22 \%$ & $53 \%$ & $\mathrm{H} / \mathrm{T}$ \\
\hline K02EC2A & 4.94 & 0.179 & 8.6 & 2452320.5 & $\mathrm{~L}_{4}$ & $27 d$ & $69 \%$ & $14 \%$ & $\mathrm{H}$ \\
\hline K02F18R & 5.45 & 0.101 & 2.0 & 2452320.5 & $\mathrm{~L}_{4}$ & $35 d$ & $43 \%$ & $20 \%$ & $\mathrm{~T}$ \\
\hline K02G31P & 5.27 & 0.273 & 3.9 & 2452360.5 & $\left(\mathrm{~L}_{4}\right)$ & $22 d$ & $3 \%$ & $6 \%$ & $\mathrm{~N}$ \\
\hline K02GG2Y & 4.99 & 0.159 & 10.4 & 2452360.5 & $\mathrm{~L}_{4}$ & $12 d$ & $100 \%$ & $95 \%$ & $\mathrm{~T}$ \\
\hline
\end{tabular}

about the same length or shorter than for the Trojans in Table 2. The error limit should therefore be larger than from an orbital fit, thus the generated clones should include the real orbit of each object.

The result of this type of random generation of orbits for $1996 \mathrm{AV}_{10}$ is shown in the bottom left panel of Fig. 5. A comparison can be made with the top left panel of Fig. 5. Despite the fact that statistically less than $2 \%$ (corresponding to the part of the new parameter space also occupied by the old. That is, $0.5^{6}$, half the length of the box side in six dimensions) of the generated clones in the bottom left panel of Fig. 5 should fulfill the criteria for a clone in the top left panel of Fig. 5 (position checked orbits); the panels have no large differences in the short-term evolution. All clones experience dynamical satellite capture and only $7 \%$ of the clones do not have a horseshoe-like orbit between the years 100 and 300 .

\section{Results}

A general conclusion for all selected objects in this study is that due to chaotic behaviour from frequent close encounters with Jupiter, the "true" orbit of these objects cannot be traced, at best, for more than a few hundred years. This also means that the long term solution of specific orbits will within a short time frame be affected by the choice of the planetary system, integration parameters and the numerical integrator used. The encounters with Jupiter do not have to be extremely close to affect the evolution of the orbital elements. Since the Tisserand parameter to Jupiter for the objects is close to 3, the encounters will have a long duration, affecting objects more strongly.

\subsection{Capture of comets}

Comets temporarily captured by Jupiter into the 1:1 mean motion resonance region appear to be common. The known population of Jupiter family comets are more than 200 (Fernández et al. 2002). Captures from other comet sources are also possible provided the objects come close enough to Jupiter. The requirement for an object to be temporarily trapped in the 1:1 mean motion resonance region is simply to have a semimajor axis close to that of Jupiter $(\approx 4.9-5.5$ AU). Of course if the semimajor axis difference is too small the object might be ejected again after one orbital period, like $2002 \mathrm{GP}_{31}$, because the critical argument has not had the time to recede enough from $0^{\circ}$ to avoid a new close encounter with Jupiter. In order to get long-lasting captures into Trojan orbits, the eccentricities generally have to be below 0.3 . All the comets in Table 1 are more likely to have an origin outside the orbit of Jupiter than inside within the integration time frame. P/2000 Y3 (Scotti) will serve as an example.

$\mathrm{P} / 2000 \mathrm{Y} 3$ is currently in a horseshoe orbit (Fig. 2, left panel) lasting for half a period. The entrance took place quite recently and is described in Lagerkvist \& Hahn (2001). $45 \%$ of the clones have a concentrated origin at a semimajor axis of around $12 \mathrm{AU}$. This is close to the 2:7 mean motion resonance with Jupiter, the 5:7 mean motion resonance with Saturn and the 2:1 mean motion resonance with Uranus. From the years -1000 to -700 the critical argument to Saturn $\sigma_{\mathrm{s}}=7 \lambda-5 \lambda_{\mathrm{s}}-2 \varpi$ oscillates around $0^{\circ}$ while the argument to Jupiter $\sigma_{\mathrm{j}}=7 \lambda-2 \lambda_{\mathrm{j}}-5 \varpi$ is close to $180^{\circ}$. For Uranus the argument $\sigma_{\mathrm{u}}=\lambda-2 \lambda_{\mathrm{u}}+\varpi$ is almost constant at $60^{\circ}$. Between -700 and -300 years none of the arguments are oscillating, but show a slow decrease, except for $\sigma_{\mathrm{u}}$ which is increasing. The slowest change is in $\sigma_{\mathrm{u}}$, followed by $\sigma_{\mathrm{j}}$ and $\sigma_{\mathrm{s}}$. The Tisserand parameter is about 2.9 to both Jupiter and Uranus, and to Saturn 2.6. The planetary encounters experienced by the clones at about the years -200 and 0 are both related to Jupiter. The other encounters by different clones in the past are totally dominated by Jupiter. There is no connection to the Trojans in the resent past.

The future development is more uncertain with no obvious preferred orbit. In this integration there are but two orbits leading to a temporary horseshoe capture. One of the orbits does not belong to the main stream seen backwards in time and the other horseshoe capture takes place after a few chaotic approaches to Jupiter. $92 \%$ of the clones have a semimajor axis larger than that of Jupiter at the end of the integration.

\subsection{High eccentricity captures}

Although Trojan orbits usually have eccentricities below about $0.2-0.3$, captures in the $1: 1$ mean motion resonance region exist for high eccentricities as well. The duration of these captures are usually only a few libration periods due to their unstable nature. However, the length of the time interval spent in the 1:1 mean motion resonance area can exceed 100000 years. The $\mathrm{L}_{4}$ and $\mathrm{L}_{5}$ points are pushed away from Jupiter from the usual location of $60^{\circ}$ and $300^{\circ}$, respectively. High eccentricity orbits are not restricted to comets, but also include asteroids. The effects of the eccentricity and the inclination on the 

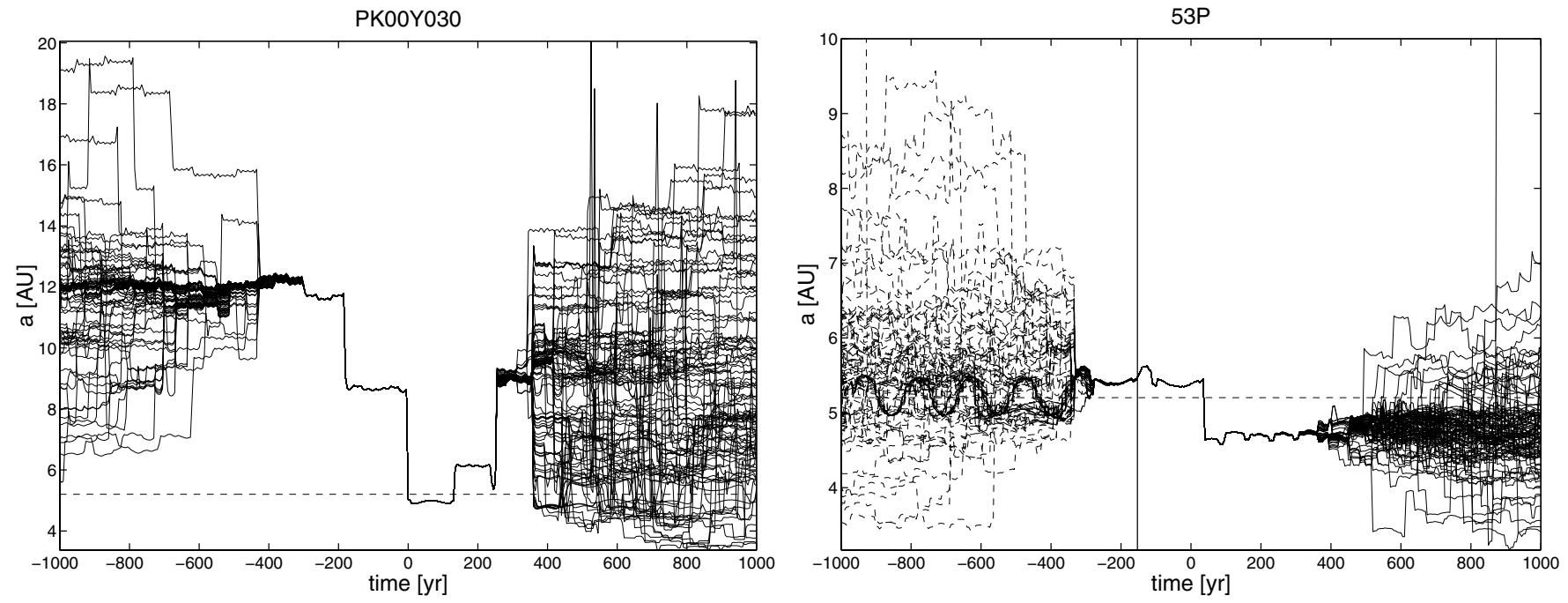

Fig. 2. Comets P/2000 Y3 (Scotti) and 53P/Van Biesbroeck. Left: evolution of P/2000 Y3 in the semimajor axis for 100 clones. The clone concentration backward in time is near three mean motion resonances: 2:7 with Jupiter, 5:7 with Saturn and 2:1 with Uranus. Both approaches between the years -200 and 0 is to Jupiter. Right: evolution in the semimajor axis for 100 clones. Before 1850 the data is not relevant due to cometary splitting. Most clones end up in the numerous high order mean motion resonance areas between the Trojans and the Hildas.

Lagrangian points have been studied by Namouni \& Murray (2000).

Comet 53P/Van Biesbroeck has an interesting sinusoidallike wave before -300 in time (right panel of Fig. 2) in the semimajor axis for a large number of the clones. But $53 \mathrm{P}$ is one of a very few number of comets that is believed to be a fragment of cometary splitting. The split of the parent object took place around 1850 prior to a close encounter with Jupiter (Carusi et al. 1985). The evolution before 1850 is therefore probably false because the other fragment from the split, 42P/Neujmin 3, does not have the same wave shape back in time. However, this is the shape of a dynamical satellite orbit (compare with top left panels of Fig. 3).

This comet is currently in a horseshoe-like orbit that will last for half a libration period. After an approach to Jupiter in $2013,53 \mathrm{P}$ will be trapped in the 7:6 resonance with Jupiter for at least 300 years. Beyond 2360 the elements start to diverge significantly but about $50 \%$ of the clones stayed between 4.4 and 5.0 AU for the remainder of the integration.

At the end of the integration only $8 \%$ of the clones were involved in the $1: 1$ resonance, $2 \%$ have dynamical satellite orbits, $4 \%$ have horseshoe orbits and $2 \%$ have tadpole orbits. These orbit types are maintained for at most a few periods mainly due to the high eccentricity (most Trojans have eccentricities lower than 0.2, which prevents long lasting Trojan orbits). At these eccentricities the $\mathrm{L}_{4}$ and $\mathrm{L}_{5}$ are pushed some $30^{\circ}$ in the mean longitude towards the $\mathrm{L}_{3}$ point. As long as the eccentricity remains high this comet can only have temporary visits in the Trojan region.

$2000 \mathrm{EJ}_{37}$ has an unusually high eccentricity for an asteroid. It is not quite a NEO, but it is a Mars crosser. One very interesting thing is that despite the high eccentricity some clones get caught in the Trojan region. The $\mathrm{L}_{4}$ and $\mathrm{L}_{5}$ are no longer located at $60^{\circ}$ from Jupiter, but for this eccentricity they are shifted some $40^{\circ}$ towards the $\mathrm{L}_{3}$ point (Fig. 3, bottom panels). In the Jupiter corotation frame, the capture into the Trojan region is almost unnoticeable. It consists of two approaches to Jupiter separated by an orbital period. The first approach is closest to Jupiter, increasing the semimajor axis to almost that of Jupiter, and the second approach takes place in the same direction as the first one (from a distance outside Jupiter) but on the opposite side of the planet, slightly reducing the semimajor axis, but not enough to detach the asteroid from the 1:1 deep resonance area.

In the top right panels of Fig. 3 a skewed horseshoe orbit can be seen where the turning point is located at $80^{\circ}$. Some clones also experience a mixing between a tadpole and a moon orbit, a skewed tadpole orbit. Outside the vertically marked areas in the bottom panels of Fig. 3 (before the year 9200 and after the year 11900) a doubled skewed tadpole orbit, including both $\mathrm{L}_{4}$ and $\mathrm{L}_{5}$, can be seen. Along with these skewed orbits and the regular LP-librations, dynamical satellite orbits are also present (similar to Fig. 3, top left panels). $4 \%$ of the clones are trapped for more than 10000 years in this type of orbit, one clone for more than 100000 years.

\subsection{Temporary dynamical satellites}

Due to the 3-body dynamics, objects can be captured in orbits around Jupiter in its corotating reference frame without having an orbit energetically bound to Jupiter. This orbit type can take place within a wide range of eccentricities. Below there are examples from two different object groups; comets and Trojans. A capture from a third group (asteroids) can be seen in the bottom panels of Fig. 4.

At the moment, comet $\mathrm{P} / 1997 \mathrm{~V} 1$ (Larsen) is in a horseshoe-like orbit (see Fig. 4, top left panel) in the $\mathrm{L}_{4}$ area moving towards $\mathrm{L}_{5}$ via $\mathrm{L}_{3}$. Around 125 years after the zero time of the integration, when the comet reaches the $\mathrm{L}_{5}$ turning point it will have a temporary capture by Jupiter leading to 11 consecutive revolutions around Jupiter lasting about 125 years. The 

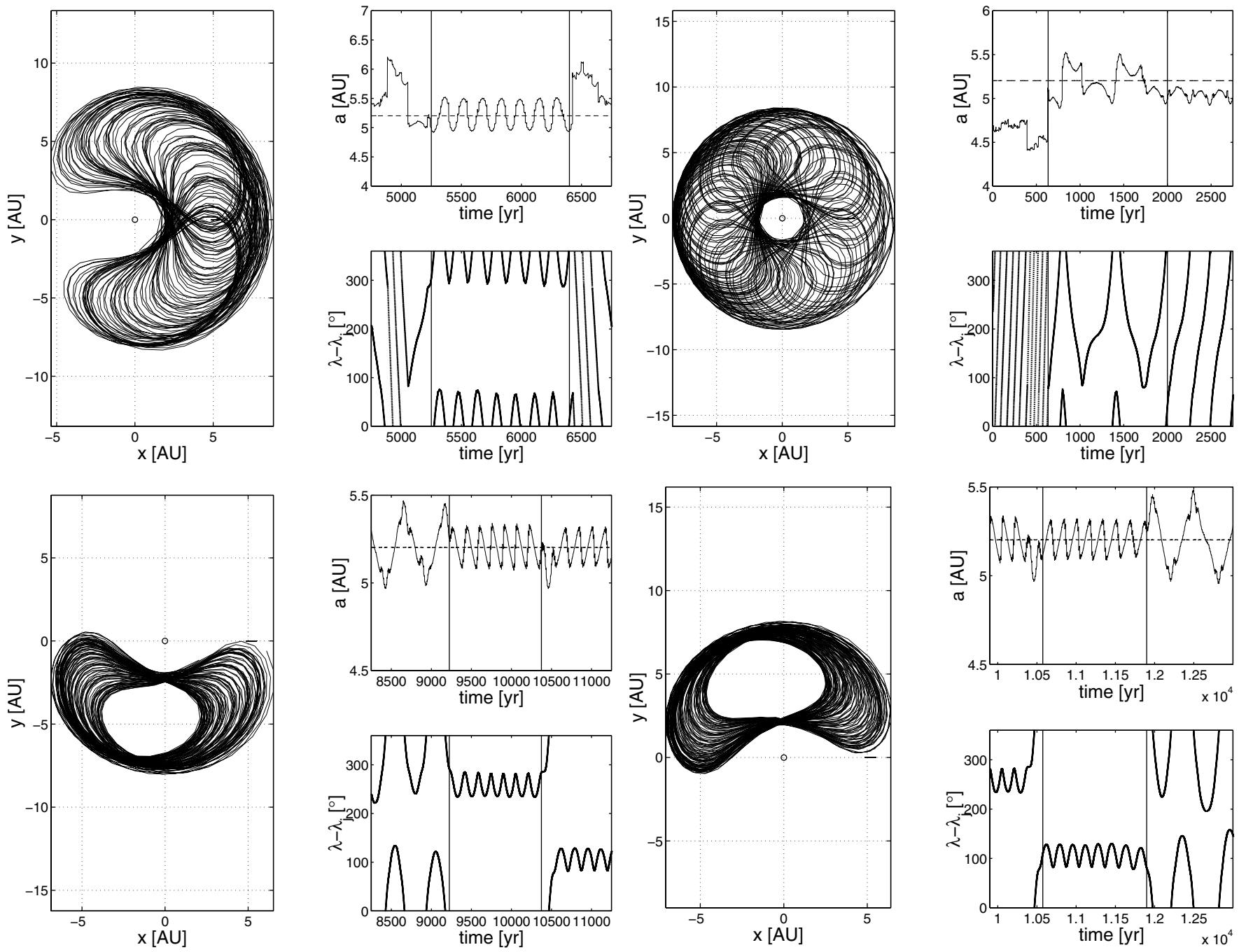

Fig. 3. High eccentricity objects in Jupiter's 1:1 mean motion resonance. Top left (3 panels): example of a dynamical satellite orbit with high eccentricity. The libration $D$ is about $130^{\circ}$. Each "bean" trajectory is close to one Jupiter year. The data between the vertical lines is shown in the Jupiter corotational system (the left of the three panels). In every case the trajectory encloses Jupiter. Top right (3 panels): an example of a skew horseshoe orbit with a libration turning point around $80^{\circ}$ from Jupiter. The data between the vertical lines in the right panels are displayed in the left panel in the Jupiter corotating system with Jupiter on the positive $x$-axis and the Sun in the origin. The actual turning point is difficult to see in the left panel since the trajectory is overlapping. Bottom left ( 3 panels): example of a high eccentricity $\mathrm{L}_{5}$ tadpole orbit. The libration point is located at a value of around $260^{\circ}$ instead of the usual $300^{\circ}$. The data between the vertical lines in the right panels are displayed in the left panel in the Jupiter corotating system with Jupiter on the positive $x$-axis (the short line at $5 \mathrm{AU}$ ) and the Sun in the origin. Bottom right (3 panels): example of a high eccentricity $\mathrm{L}_{4}$ tadpole orbit. The libration point is located at a value of around $100^{\circ}$ instead of the usual $60^{\circ}$. The data between the vertical lines in the right panels are displayed in the left panel in the Jupiter corotating system with Jupiter on the positive $x$-axis (the short line at $5 \mathrm{AU}$ ) and the Sun in the origin.

capture is not a satellite orbit, since the orbital energy with respect to Jupiter is positive, but a dynamical satellite orbit.

The clones start to diverge after about 450 years both backward and forward in time. Backward in time, $40 \%$ of the clones go through one whole horseshoe period between the years -450 and -750 , but only $20 \%$ continue to have more than one horseshoe period. If the clones with Trojan type orbits are followed further back in time, $4 \%$ have Trojan type orbits for more than 5000 years.

As discussed in Sect. 2, due to the chaotic behaviour of close approaches with Jupiter and the large number of them, specific orbits cannot be fully reproduced, down to the given number of decimals in the orbital elements, after a few thousand years. This mean that it cannot be concluded if any of the orbits are stable backwards in time, but the possibility exists that comet P/1997 V1 actually is an escaped Trojan. If not, the most likely resent past is an orbit outside the orbit of Jupiter.

Forward in time, most clones end with orbits outside of that of Jupiter. Those clones that are in the Trojan area at the end of the integration time interval are all in horseshoe orbits.

$2000 \mathrm{HR}_{24}$ is also an example of an object being temporarily trapped by Jupiter in a dynamical satellite orbit (Fig. 4, bottom panels). This will occur around 2055 and lasts for 7 revolutions. The entry take place from the $\mathrm{L}_{5}$ side and the exit at the $\mathrm{L}_{4}$ side. This object is listed as a Trojan but the orbit is 

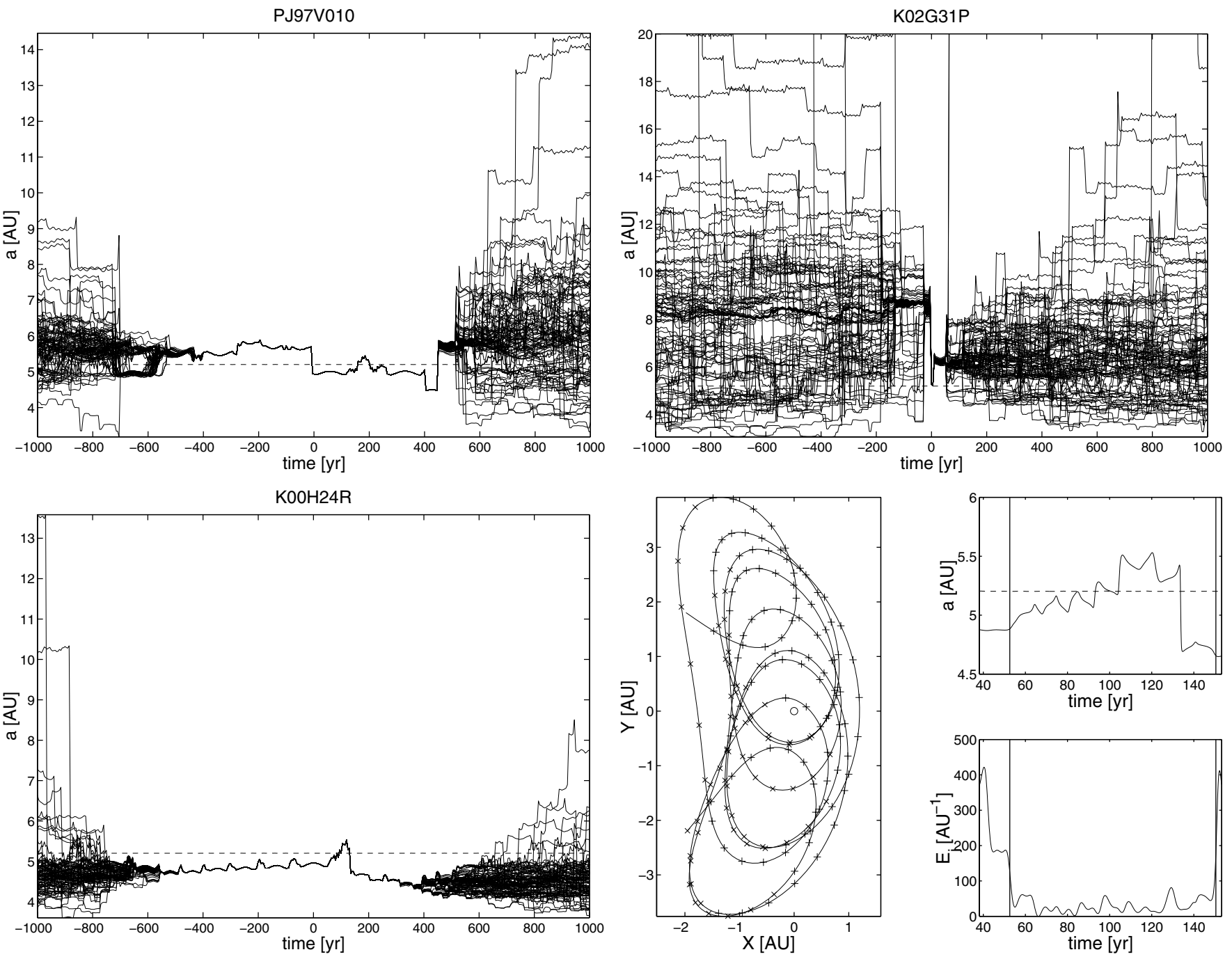

Fig. 4. Misidentified Trojans and temporary dynamical satellites. Top left: evolution of P/1997 V1 in the semimajor axis for 100 clones. There is a small chance for a Trojan origin. If so, this comet could provide a proof of ice in the interior of Trojans. Top right: evolution of $2002 \mathrm{GP}_{31}$ in the semimajor axis for 100 clones. The object does not have a Trojan orbit. It is probably a misclassified comet. Bottom left: evolution of $2000 \mathrm{HR}_{24}$ in the semimajor axis for 100 clones. This object is actually not a Trojan as clearly can be seen. The evolution of the semimajor axis is not unlike both (6144) and (32511). Bottom right (3 panels): dynamical satellite capture for seven revolutions of $2000 \mathrm{HR}_{24}$ around Jupiter. The orbital energy to Jupiter is positive during the event (bottom right). The time between the vertical lines in the right panels are displayed to the left in the jovocentric corotating system with Jupiter at the origin (circle) and the Sun on the negative $x$-axis. $(+)$ are above the orbital plane of Jupiter and (x) are below the plane. Time between the marks is 200 days.

not that of a Trojan. Actually only one clone gets trapped in a Trojan orbit at the end of the integration. $78 \%$ of the clones both start and end at a semimajor axis lower than Jupiter's, mostly between 4 and 5 AU. $14 \%$ of the clones have temporary Jupiter moon captures also backwards in time. The evolution of the semimajor axis is not unlike that of (6144) and (32511). However, $2000 \mathrm{HR}_{24}$ differs from the other two object in that the inclination is higher and the eccentricity is lower.

The Trojan asteroid $1996 \mathrm{AV}_{10}$ is currently in a horseshoelike orbit and is on the opposite side of the Sun as seen from Jupiter (Fig. 5, top left panel). It is now travelling from the $\mathrm{L}_{4}$ area to the $\mathrm{L}_{5}$ area. Similar to $\mathrm{P} / 1997 \mathrm{~V} 1$, this Trojan will have a dynamical satellite orbit for 3 retrograde revolutions around the year 2050. The capture is initiated from the $\mathrm{L}_{5}$ side and ends on the $\mathrm{L}_{4}$ side. But the critical argument $\sigma=\lambda-\lambda_{\mathrm{j}}$ does not cross $0^{\circ}$ more than once, so this event can be seen more or less as an accidental bypass rather than a real capture.

Backwards in time there are no Trojan orbits lasting the whole integration, which suggests that this asteroid was captured around 1948 from a semimajor axis larger than that of Jupiter's. Forward in time, $17 \%$ of the clones have horseshoe orbits lasting between 1000 and 3500 years. Two additional clones have horseshoe orbits lasting more than 8000 years. The 14 day arc is short but this Trojan was discovered in more or less the same sky area relative to Jupiter as it is in today, which means that the combination of the orbital elements $\Omega, \omega$ and $M$ may vary several degrees without a significant change in the resulting Trojan orbit. The semimajor axis is at this stage more restricted, if a horseshoe orbit is to be maintained. A deviation of more than $0.05 \mathrm{AU}$ from the nominal value in Table 1 will 

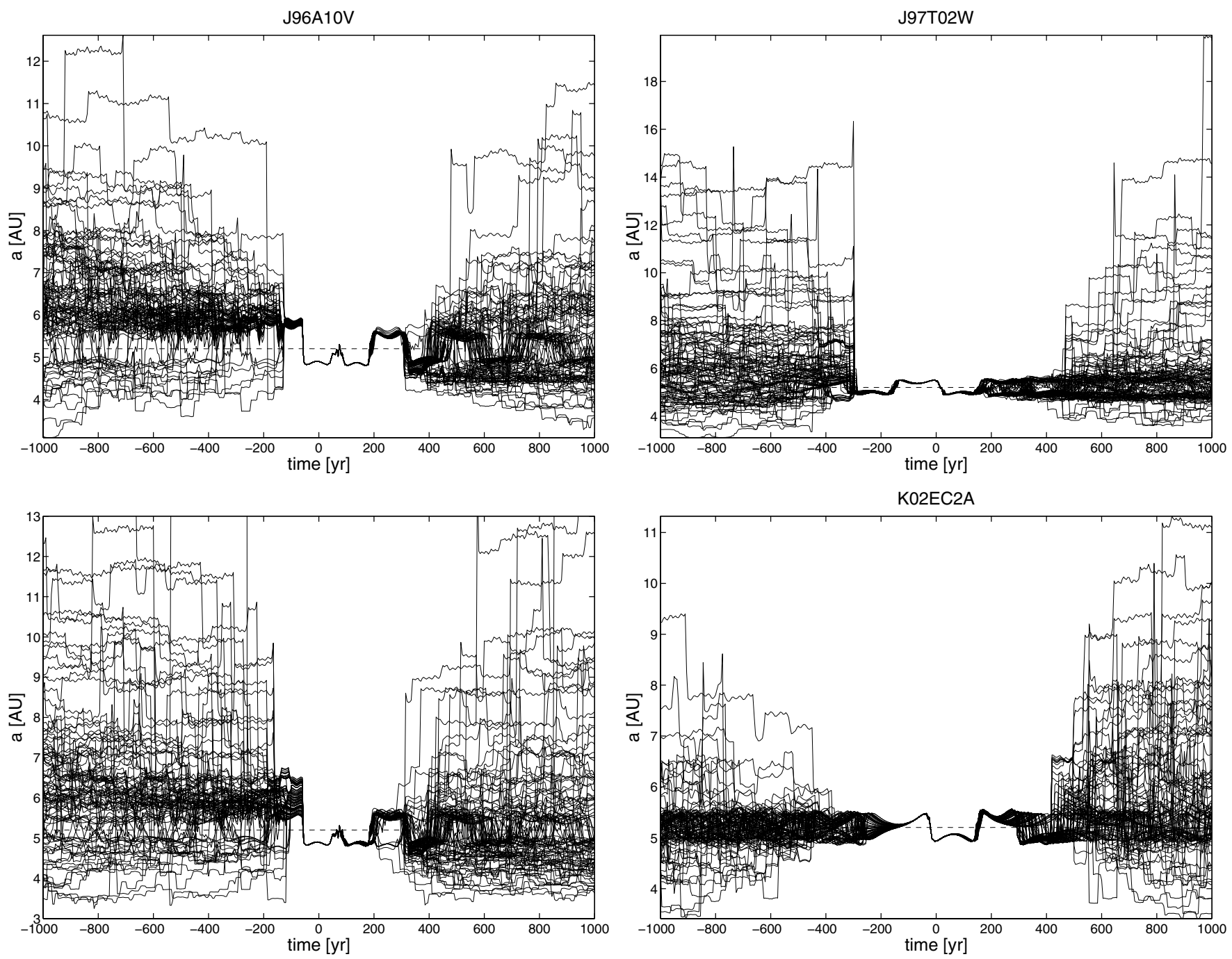

Fig. 5. Trojans $1996 \mathrm{AV}_{10}, 1997 \mathrm{TW}_{2}$ and $2002 \mathrm{EA}_{122}$. Top left: evolution of $1996 \mathrm{AV}_{10}$ in the semimajor axis for 100 clones. At the next approach to Jupiter it will orbit Jupiter three times. Top right: evolution of $1997 \mathrm{TW}_{2}$ in the semimajor axis for 100 clones. Despite a short observational arc it is not unlikely that this Trojan is in a horseshoe orbit since it was observed relatively close to Jupiter. Bottom left: a comparison to the top left panel but with a random difference in the orbital elements twice that used for $1996 \mathrm{AV}_{10}$. The same uncertainty is used for all objects in Table 2. The general look from the top left panel is preserved, the dynamical satellite capture included. Bottom right: evolution of $2002 \mathrm{EA}_{122}$ in the semimajor axis for 100 clones. This Trojan is the best candidate for an ejection within the next 1000 years from the Trojan area. Currently it is in a horseshoe orbit.

certainly jeopardise the existence of the horseshoe-like orbit shown in the top left of Fig. 5.

\subsection{Trojans}

In the diffusion process of Trojans they generally pass through the horseshoe orbit before finally being ejected from the Trojan swarms by close passages to Jupiter. The maximum amplitude allowed in the semimajor axis depends on the other orbital elements. A larger semimajor axis amplitude corresponds to a larger libration. A low eccentricity Trojan can therefore have a larger semimajor axis amplitude than a high eccentricity Trojan without risk of being ejected.

The ejection of a Trojan is not necessarily a straightforward process (tadpole-horseshoe-non-Trojan) but can go between a tadpole and a horseshoe orbit several times. An ejected Trojan can also soon after the escape be temporary recaptured. Direct ejection from a tadpole orbit is also possible if the eccentricity is high enough.

The listed Trojan $1997 \mathrm{TW}_{2}$ has only a 2 day observational arc. However, since the nominal orbit currently gives a horseshoe solution (Fig. 5, top right panel) and the horseshoe orbit is not extremely sensitive to the eccentricity, it has been included in this study. About $50 \%$ of the clones in both the backward and forward part of the integration have semimajor axes larger than that for Jupiter. Backwards in time $10 \%$ of the clones have a Trojan orbit during the integration and forward in time the number is $7 \%$, but only $2 \%$ of the clones have Trojan orbits during the whole integration time span. The longest lasting Trojan orbit backwards lasts for 6800 years, but as described earlier the number of approaches to Jupiter makes it uncertain if any of 
the clones can be stable backwards. Forward in time the longest lasting orbit is stable for 5000 years.

The most probable outcome for this type of object with a short observational arc is that in the end, they will turn out to be tadpole objects when more observations are available. However, this object was discovered relatively close to Jupiter which increases the chance that it actually is a horseshoe Trojan.

$2002 \mathrm{EA}_{122}$ (Fig. 5, bottom right panel) is currently the best Trojan candidate for being ejected during the next 1000 years. Many of the clones are going back and forth between tadpole and horseshoe orbits, but $31 \%$ of the clones have Trojan orbits during the whole integration interval.

$2002 \mathrm{GP}_{31}$ is, like $2000 \mathrm{HR}_{24}$, a misidentified Trojan. The narrow dip in the semimajor axis in the top right panel of Fig. 4 (close to year 0) is about twelve years long and it comes from the annual movement of the object. This object came close to Jupiter during 1998 (probably from the 1:2 mean motion resonance with Jupiter), and had the semimajor axis reduced to almost 5.2 AU. Since the mean anomaly was close to $0^{\circ}$ at this time and the eccentricity is larger than for Jupiter the object turned towards the $\mathrm{L}_{4}$ cloud in the Jupiter corotating system. The mean anomaly is increasing, so the relative velocity away from Jupiter will be reduced and the object is eventually going to turn back towards Jupiter until it, one revolution after the first encounter, again is near Jupiter (from a larger solar distance than Jupiter). This time the semimajor axis will be increased beyond the borders of the 1:1 mean motion resonance with Jupiter.

A total of $18 \%$ of the clones have an origin in the $1: 2$ mean motion resonance with Jupiter in this integration. This, and the overall picture in Fig. 4 (top right panel), suggests that $2002 \mathrm{GP}_{31}$ is a comet. Only a few clones have an origin (3\%) and an end (6\%) in Trojan orbits.

\section{Discussion}

Using randomly distributed elements within an error box for the clones (as in Table 2) is a very fast way to produce orbits enclosing the orbit of the real object compared to performing a check of each clone against observed positions (as in Table 1). Although only a small portion of the former type of clones fit within the error of the observed positions, the evolution of the elements are not strongly affected within a short time frame. But in order to minimise the number of false clones, a few steps can be taken. First, for each object the individually determined orbital element errors should be used. Second, the orbital elements are not totally independent of each other. Usually simple constraints can be found from different combinations of the elements. This will smooth the edges of the error box. The extent of the time frame for which the orbits of the "error box" generated clones, and the "position verified" clones produce the same general result are of course dependent on the perturbing environment.

Since the same error limits are used for the objects in Table 2, but the observational arcs for some of them are significantly longer than for $1996 \mathrm{AV}_{10}$, the diffusion of clones should be smaller. The resulting evolution in the semimajor axis can therefore be seen as an outer boundary. Although the initially generated clones came from an orbital element space slightly larger than those that fitted observations, it cannot be excluded that among the objects in Table 1, there can still be a few more possible orbital element sets outside the space occupied by the clones. This would mean that the boundaries in the semimajor axis evolution are lower limits.

Among the objects presented in this paper, four are likely to be transitional objects between stable tadpole orbits and escaped Trojans. Several of the objects in the semimajor axis interval (4.6-5.8 AU) are in temporary Trojan orbits both today and in the near past or future. There are also some objects experiencing dynamical satellite captures.

If the comet $\mathrm{P} / 1997 \mathrm{~V} 1$ is an escaped Trojan, it is probably a collision fragment. No ice has yet been detected on any Trojan although it can be expected to exist (e.g. Hartmann et al. 1987; Hartmann \& Tholen 1990; Marzari et al. 1995), so confirming a Trojan origin of this comet could provide direct proof of ice in the interior of the Trojans. Tancredi et al. (2000) give an estimated size of $3.6 \mathrm{~km}$ and an absolute magnitude (nuclear) of $H=14.8$. For a corresponding Trojan, the visual magnitude at opposition would be around 21.5 . This is below the detection limit for the survey telescopes used today which means that $\mathrm{P} / 1997 \mathrm{~V} 1$ can be either the main body or a large fragment from a collision. A main body is the most probable scenario. Firstly, because there are no other comets in the same orbit. $\mathrm{P} / 1999 \mathrm{WJ}_{7}$ looks similar in the semimajor axis in the resent past, but the closest distance to $\mathrm{P} / 1997 \mathrm{~V} 1$ was about $0.3 \mathrm{AU}, 50$ years ago, and not far from the $\mathrm{L}_{3}$ point. Secondly, there is no indication of any recently formed collisional family in the Trojan swarms. If $\mathrm{P} / 1997 \mathrm{~V} 1$ is the main body of a smaller none-disruptive collision the other fragments should be too faint to detect, and hence, only one object is seen.

Lagerkvist et al. (2002) found no difference between the $\mathrm{L}_{4}$ and $\mathrm{L}_{5}$ swarms except for the inclination. $\mathrm{L}_{5}$ is deficient around an inclination of $8-10^{\circ}$ compared to $\mathrm{L}_{4}$. Could it be a bias effect? The dedicated Trojan surveys made before 1998 are mostly of the $\mathrm{L}_{4}$ area (see Sect. 1). These surveys have generally a deeper limiting magnitude than the NEO surveys, making it possible for more low inclination detections than today. But if the observations are split into before 1998 and after 1997 the same deficiency in $\mathrm{L}_{5}$ around an inclination of $8-10^{\circ}$ remains. Moreover, there are still $50 \%$ more detections in $\mathrm{L}_{4}$ than in $\mathrm{L}_{5}$. This suggests that the inclination difference and number difference is real and not a bias effect.

In the classical 3-body problem there are no differences between the two LPs. The differences between the swarms should therefore be searched for in the early solar system. In the solar nebula, adding the effect of gas, the symmetric picture of the LPs changes. Peale (1993) has investigated the effect on the LP of gas drag. The LPs should have been shifted so that $\mathrm{L}_{4}$ was pushed away from Jupiter while $\mathrm{L}_{5}$ was located closer to Jupiter. This would favour stability in the $\mathrm{L}_{5}$ and hence more $\mathrm{L}_{5}$ Trojans. Marzari \& Scholl (1998) came to the same conclusion. This does not appear to be correct from the observation of Jupiter Trojans. However, for Mars this is the case, with 5 of 6 Trojans in the $\mathrm{L}_{5}$ swarm. The difference between theory and observation is still unresolved. 
From the semimajor axis evolution in Fig. 4 (top right panel), $2002 \mathrm{GP}_{31}$ appears to be a comet. Extending the integration backward for clones with an origin in the 1:2 mean motion resonance with Jupiter points to an origin in the outer solar system. But as far as 10000 years back in time the perihelion distances are still close to Jupiter. The object is currently about as bright as it gets but still fainter than the 21st magnitude. With a large telescope it should not be difficult to locate it, and check for a coma. Images taken with the Danish $1.54 \mathrm{~m}$ telescope at La Silla on 9th February 2003 ( $R$-magnitude $\approx 21.7$ ) by G. Masi and R. Michelsen showed no sign of a coma (Michelsen 2003).

A large portion of the asteroids ( 3 of 10) and the comets (5 of 8 ) had temporary captures in the 1:1 mean motion resonance area during the 2000 year period of the integration. This suggests that there are several more candidates for temporary captures among the objects with semimajor axes just outside the interval selected in this study, especially in the Jupiter family comet group. For instance, both 82P/Gehrels 3 (Rickman $\&$ Malmort 1981) and 111P/Helin-Roman-Crockett (Tancredi et al. 1990) with reported capture events have semimajor axes of 4.13, and 4.05 AU respectively. Also, the comet fragments of D/Shoemaker-Levy 9 have not been considered partly due to the very complex trajectory (Kary \& Dones 1996) prior to the collision with Jupiter.

All of the Trojans in Table 1 and most of the Trojans in Table 2 have orbits with short observational arcs. Those with observational arcs of only a few days will most likely eventually be identified as tadpole Trojans. But in order to better determine the orbits they must either be precovered by findings in archive material or recovered by new observations.

Acknowledgements. I would like to thank B. Davidsson, C.-I. Lagerkvist and J. Warell for reading the manuscript and suggesting improvements. I would also like to thank A. Gnädig for orbit determinations and G. Masi \& R. Michelsen for their effort to detect a coma around $2002 \mathrm{GP}_{31}$. During the work I was financially supported by Anna \& Allan Löfberg's fund.

\section{References}

Carusi, A., Kresák, L., Perozzi, E., \& Valsecchi, G. B., in Dynamics of Comets: Their Origin and Evolution, ed. A. Carusi, G. B. Valsecchi (Holland: D. Reidel publishing company), 319

Fernández, J. A., Gallardo, T., \& Brunini, A. 2002, Icarus, 159, 358

Hartmann, W. K., \& Tholen, D. J. 1990, Icarus, 86, 448

Hartmann, W. K., Tholen, D. J., \& Cruikshank, D. P. 1987, Icarus, 69, 33

Kary, D. M., \& Dones, L. 1996, Icarus, 121, 207

Lagerkvist, C.-I., \& Hahn, G. 2001, AN, 322, 265

Lagerkvist, C.-I., Mottola, S., Carsenty, U., et al. 2000, in Minor Bodies in the Outer Solar System, ed. A. Fitzsimmons, D. Jewitt, \& R. M. West (Germany: Springer), 179

Lagerkvist, C.-I., Karlsson, O., Hahn, G., et al. 2002, AN, 323, 475

Levison, H. F., \& Duncan M. J. 1994, Icarus, 108, 18

Levison, H. F., Shoemaker, E. M., \& Shoemaker, C. S. 1997, Nature, 385,42

Marsden, B. G. (ed.) 1987, M.P.C., 12493

Marsden, B. G. (ed.) 1989, M.P.C., 14819

Marsden, B. G. (ed.) 1991, M.P.C., 19055

Marzari, F., \& Scholl, H. 1998, Icarus, 131, 41

Marzari, F., \& Scholl, H. 2002, Icarus, 159, 328

Marzari, F., Farinella, P., \& Vanzani, V. 1995, A\&A, 299, 267

Michelsen, R. 2003, Private Communication

Namouni, F., \& Murray, C. D. 2000, Cel. Mech. Dyn. Astron., 76, 131

Peale, S. J. 1993, Icarus, 106, 308

Rickman, H., \& Malmort, A. M. 1981, A\&A, 102, 165

Shoemaker, E., Shoemaker, C. S., \& Wolfe, R. F. 1989, in Asteroids II, ed. R. P. Binzel, T. Gehrels, M. Shapley Matthews (Tucson: The University of Arizona Press), 487

Tancredi, G., Lindgren, M., \& Rickman, H. 1990, A\&A, 239, 375

Tancredi, G., Fernández, J. A., Rickman, H., \& Licandro, J. 2000, A\&AS, 146,73

Van Houten, C. J., Van Houten-Groeneveld, I., \& Gehrels, T. 1970a, AJ, 659

Van Houten, C. J., Van Houten-Groeneveld, I., Herget, P., \& Gehrels, T. 1970b, A\&AS, 2, 339

Wisdom, J., \& Holman, M. 1991, AJ, 102, 1528 